\title{
DISCRETE WICK CALCULUS AND STOCHASTIC FUNCTIONAL EQUATIONS ${ }^{+}$
}

by

\author{
Helge Holden ${ }^{*}$, Tom Lindstrøm ${ }^{* *}$, Bernt $\emptyset \mathrm{ksendal}^{* *}$, and Jan Ubøe $\mathrm{e}^{* * *}$
}

\section{INTRODUCTION}

The purpose of this paper is to work out the relationship between white noise analysis and ordinary calculus in as simple a setting as possible. By studying Wick calculus over finite probability spaces, we try to focus on fundamental algebraic relations which are easy to lose sight of in the complicated analytic setting of the continuous theory. Our approach is very much influenced by P.A. Meyer's "Toy Fock space" version of quantum probability theory (see, e.g. [5], [6]).

In the first section of the paper we work out the basic properties of Wick products on toy Fock spaces. If $f\left(x_{1}, \cdots, x_{n}\right)$ is an analytic function and $X_{1}, X_{2}, \cdots, X_{n}$ are random variables, we define the Wick functional $f^{\diamond}\left(X_{1}, \cdots, X_{n}\right)$ by replacing all ordinary products in the power series of $f$ by Wick products. By an easy computation we determine the Walsh decomposition of $f^{\diamond}\left(X_{1}, \cdots, X_{n}\right)$ in terms of the Walsh decompositions of $X_{1}, \cdots, X_{n}$.

In Section 2 we use this relationship to prove the main theorem of the paper: Assume that $f: \mathbb{C}^{n} \times \mathbb{C}^{k} \rightarrow \mathbb{C}^{n}$ is an analytic function and that $Y_{1}, Y_{2}, \cdots, Y_{k}$ are given random variables with expectations $y_{1}, \cdots, y_{k}$. Then (under a non-degeneracy condition) there is a one-to-one correspondence between solutions $x_{1}, \cdots, x_{n}$ of the equation

$$
f\left(x_{1}, \cdots, x_{n}, y_{1}, \cdots, y_{k}\right)=0
$$

and solutions $X_{1}, \cdots, X_{n}$ of the equation

$$
f^{\diamond}\left(X_{1}, \cdots, X_{n}, Y_{1}, \cdots, Y_{k}\right)=0 .
$$

Moreover, given a solution $x_{1}, \cdots, x_{n}$ of the first equation, the corresponding solution of the second equation can be found by solving a hierarchy of linear equations.

In the last two sections we take a brief look at possible applications - first to ordinary stochastic differential equations where the coefficients are Wick functionals, and then to

+ This research is supported by VISTA, a research cooperation between the Norwegian Academy of Science and Letters and Den Norske Stats Oljeselskap a.s. (STATOIL)

* Division of Mathematical Sciences, Norwegian Institute of Technology, University of Trondheim, N-7034 Trondheim, Norway

** Department of Mathematics, University of Oslo, P.O. Box 1053, Blindern, N-0316 Oslo 3, Norway

*** Haugesund Maritime College, Skåregaten 103, N-5500 Haugesund, Norway 
partial differential equations with a stochastic term. Of course, within the discrete theory developed here we can only treat discrete approximations to these problems, but the methods still give a strong indication of what a continuous approach might lead to. And this brings us to the question of whether it is possible to formulate a similar principle in a continuous setting; a question we shall not attempt to answer here, but which we hope to return to in the future.

Readers who want to know more about (continuous) white noise analysis, are referred to the forthcoming book by Hida, Kuo, Potthoff, and Streit [1].

\section{Wick calculus on toy Fock spaces}

Fix a finite set $\Lambda$. Let

$$
\Omega=\{\omega \mid \omega: \Lambda \rightarrow\{-1,1\}\}
$$

be the set of all Bernoulli trials over $\Lambda$, and give $\Omega$ the uniform probability measure $P$. We shall sometimes assume that we are given a measure $\mu$ on $\Lambda$, and refer to the function $W: \mathcal{P}(\Lambda) \times \Omega \rightarrow \mathbb{R}$ defined by

$$
W(A, \omega)=\sum_{a \in A} \omega(a) \sqrt{\mu(a)}
$$

as white noise over $\Lambda$. Although $\mu$ and $W$ are not really needed for the systematic development of our theory, we shall occasionally use them to facilitate comparison to the continuous theory.

By toy Fock space over $\Lambda$ we shall simply mean the space $L^{2}(\Omega, P)$; i.e., the set of all functions $X: \Omega \rightarrow \mathbb{C}$ with the norm

$$
\|X\|=\left(\sum|X(\omega)|^{2} P(\omega)\right)^{\frac{1}{2}}
$$

For each $A \subset \Lambda$ define a function $\chi_{A}: \Omega \rightarrow \mathbb{C}$ by

$$
\chi_{A}(\omega)= \begin{cases}\prod_{a \in A} \omega(a) & \text { if } A \neq \emptyset \\ 1 & \text { if } A=\emptyset\end{cases}
$$

Clearly, $\left\{\chi_{A}: A \subset \Lambda\right\}$ is an orthonormal set in $L^{2}(\Omega, P)$ and since its cardinality equals the dimension of $L^{2}(\Omega, P)$, it must be a basis. Hence any function $X \in L^{2}(\Omega, P)$ can be written uniquely as a sum

$$
X=\sum_{A \subset \Lambda} X(A) \chi_{A}
$$

where each $X(A)$ is a complex number. We shall refer to this as the Walsh decomposition of $X$ - it is the discrete analogue of Wiener-Itô. chaos. 
We are now ready to introduce the Wick product on $L^{2}(\Omega, P)$. Intuitively the idea is that we want an algebraic operation $\diamond$ such that

$$
\chi_{A} \diamond \chi_{B}= \begin{cases}\chi_{A \cup B} & \text { if } A \cap B=\emptyset \\ 0 & \text { otherwise }\end{cases}
$$

If we extend by usual algebraic rules, we see that if $X=\sum X(A) \chi_{A}$ and $Y=\sum Y(B) \chi_{B}$, then $Z=X \diamond Y$ should have Walsh decomposition $Z=\sum Z(C) \chi_{C}$ where

$$
Z(C)=\sum\{X(A) Y(B) \mid A \cup B=C, A \cap B=\emptyset\}
$$

and this is what we take to be the official definition of the Wick product.

Remark: Note that if $U$ is the ordinary product of $X$ and $Y$, then $U$ has Walsh components

$$
U(C)=\sum\{X(A) Y(B) \mid A \Delta B=C\}
$$

Hence in one sense the Wick product is simpler than the ordinary product; to compute $Z(C)$ we only need to know the Walsh components $X(A)$ and $Y(B)$ for subsets of $C$, while $U(C)$ depends on all Walsh component of $X$ and $Y$. The strategy of this paper will be to exploit this feature of Wick multiplication systematically.

1.1 Example. An element $X \in L^{2}(\Omega, P)$ is called a stochastic integral if it is of the form

$$
X=\int X(s) d W(s) \equiv \sum X(s) W(s)
$$

Using that $W(s)=\sqrt{\mu(s)} \chi(s)$, the Wick product of two stochastic integrals is easily computed:

$$
\begin{aligned}
& \left(\int X(s) d W(s)\right) \diamond\left(\int Y(s) d W(s)\right)= \\
& \sum X(s) \sqrt{\mu(s)} \chi(s) \diamond \sum Y(s) \sqrt{(\mu(s)} \chi(s) \\
& =\int X(s) d W(s) \cdot \int Y(s) d W(s)-\int X(s) Y(s) d \mu(s)
\end{aligned}
$$

Our first result is a trivial observation.

1.2 Lemma: $\left(L^{2}(\Omega, P),+, \diamond\right)$ is a commutative ring with unit element $\chi_{\emptyset}=1$

With a well-defined Wick product it is straightforward to define Wick polynomials. If $p\left(z_{1}, \cdots, z_{k}\right)=\sum_{|\alpha|=0}^{m} c_{\alpha} z^{\alpha}$ is a complex polynomial in $k$ variables (where $\alpha=\left(\alpha_{1}, \alpha_{2}, \cdots, \alpha_{k}\right)$ run over multi-indices $)$, we define an associated operator

$$
p^{\diamond}: L^{2}(\Omega) \times \cdots \times L^{2}(\Omega) \rightarrow L^{2}(\Omega)
$$




$$
p^{\diamond}\left(X_{1}, \cdots, X_{k}\right)=\sum_{|\alpha|=0}^{m} c_{\alpha} X_{1}^{\diamond \alpha_{1}} \diamond \cdots \diamond X_{k}^{\diamond \alpha_{k}}
$$

where, of course, $X^{\diamond n}=X \diamond \cdots \diamond X$ ( $n$ times) for every positive integer $n$, and $X^{\diamond 0}$ is the constant 1 .

Remark: Observe that it doesn't make sense to write $p^{\diamond}(X(\omega))$ for $p^{\diamond}(X)(\omega)$; the value of $p^{\diamond}(X)$ at $\omega$ depends on the whole random variable $X$ and not only on its value at $\omega$.

In order to express the Walsh decomposition of $p^{\circ}\left(X_{1}, \cdots, X_{k}\right)$ in terms of the Walsh decompositions of $X_{1}, \cdots, X_{k}$, it is convenient to introduce the following notation. If $\alpha=\left(\alpha_{1}, \cdots, \alpha_{k}\right)$ is a multi-index and $A$ is a subset of $\Lambda$, let $\mathcal{P}_{\alpha}(A)$ be the family of all sequences of sets

$$
\left\langle\left\{A_{1}^{(1)}, A_{2}^{(1)}, \cdots, A_{\alpha_{1}}^{(1)}\right\},\left\{A_{1}^{(2)}, \cdots, A_{\alpha_{2}}^{(2)}\right\}, \cdots,\left\{A_{1}^{(k)}, \cdots, A_{\alpha_{k}}^{(k)}\right\}\right\rangle
$$

where the sets $A_{j}^{(i)}$ are nonempty, disjoint subsets of $A$ whose union is $A$. If $\alpha_{m}=0$, we just let $\left\{A_{1}^{(m)}, \cdots, A_{\alpha m}^{(m)}\right\}$ be the empty set. We shall write $\left\langle\left\{A_{j}^{(i)}\right\}>\right.$ as an abbreviation of $\left\langle\left\{A_{1}^{(1)}, \cdots, A_{\alpha_{1}}^{(1)}\right\}, \cdots,\left\{A_{1}^{(k)}, \cdots, A_{\alpha_{k}}^{(k)}\right\}>\right.$.

The zeroth order component $X_{i}(\emptyset)$ in the Walsh expansion $X_{i}=\sum X_{i}(A) \chi_{A}$ will play a particularly important part in the theory, and when it is convenient we shall denote it simply by $x_{i}$; hence $X_{i}=x_{i}+\sum_{A \neq \emptyset} X_{i}(A) \chi_{A}$.

1.3 Proposition. Let $p(z)=p\left(z_{1}, \cdots, z_{k}\right)=\sum c_{\alpha} z^{\alpha}$ be a complex polynomial in $k$ variables, and let $X_{1}, \cdots, X_{k}$ be elements in $L^{2}(\Omega)$ with Walsh decomposition

$$
X_{i}=x_{i}+\sum_{A \neq \emptyset} X_{i}(A) \chi(A)
$$

Then the Walsh decomposition $p^{\diamond}\left(X_{1}, \cdots, X_{k}\right)=\sum h(A) \chi(A)$ is given by

$$
h(\emptyset)=p\left(x_{1}, \cdots, x_{k}\right)
$$

and - for $A \neq \emptyset$

$$
\begin{aligned}
& h(A)=\sum_{\alpha} \sum_{<\left\{A_{j}^{(i)}\right\}>\in \mathcal{P}_{\alpha}(A)} D_{\alpha} p\left(x_{1}, \cdots, x_{k}\right) X_{1}\left(A_{1}^{(1)}\right) \cdot \ldots \cdot X_{1}\left(A_{\alpha_{1}}^{(1)}\right) \\
& \cdot \ldots \cdot X_{k}\left(A_{1}^{(k)}\right) \cdot \ldots \cdot X_{k}\left(A_{\alpha_{k}}^{(k)}\right)
\end{aligned}
$$

where $D_{\alpha}=\frac{\partial^{\alpha_{1}+\alpha_{2}+\cdots+\alpha_{k}}}{\partial z_{1}^{\alpha_{1}} \partial z_{2}^{\alpha_{2} \cdots \partial z_{k}^{\alpha_{k}}}}$ 
Proof: It is an elementary fact from algebra that if $p\left(X_{1}, \cdots, X_{k}\right)=\sum c_{\alpha} X^{\alpha}$ is a complex polynomial over a commutative ring $R$, then for any elements $a_{1}, \cdots, a_{k} \in R$

$$
p\left(X_{1}, \cdots, X_{k}\right)=\sum_{k} \frac{D_{\alpha} p\left(a_{1}, \cdots, a_{k}\right)}{\alpha_{1} ! \cdots \alpha_{k} !}\left(X_{1}-a_{1}\right)^{\alpha_{1}} \cdot \ldots \cdot\left(X_{k}-a_{k}\right)^{\alpha_{k}}
$$

If we apply this in our situation with $a_{i}=x_{i}$ we get

$$
p^{\diamond}\left(X_{1}, \cdots, X_{k}\right)=\sum_{\alpha} \frac{D_{\alpha} p\left(x_{1}, \cdots, x_{k}\right)}{\alpha_{1} ! \cdot \ldots \cdot \alpha_{k} !}\left(X_{1}-x_{1}\right)^{\diamond \alpha_{1}} \diamond \cdots \diamond\left(X_{k}-x_{k}\right)^{\diamond \alpha_{k}} .
$$

From the definition of the Wick product we have

$$
\begin{aligned}
& \left(X_{1}-x_{1}\right)^{\diamond \alpha_{1}} \diamond\left(X_{2}-x_{2}\right)^{\diamond \alpha_{2}} \diamond \cdot \ldots \cdot \diamond\left(X_{k}-x_{k}\right)^{\diamond \alpha_{k}} \\
& =\left(\sum_{A \neq \emptyset} X_{1}(A) \chi_{A}\right)^{\diamond \alpha_{1}} \diamond \cdots \diamond\left(\sum_{A \neq \emptyset} X_{k}(A) \chi_{A}\right)^{\diamond \alpha_{k}} \\
& =\sum_{A} \alpha_{1} ! \alpha_{2} ! \cdots \alpha_{k} ! \sum_{<\left\{A_{j}^{(i)}\right\}>\in \mathcal{P}_{\alpha}(A)} X_{1}\left(A_{1}^{(1)}\right) \cdot \ldots \cdot X_{k}\left(A_{\alpha k}^{(k)}\right) \chi(A),
\end{aligned}
$$

and substituting this into (1.8) we prove the proposition.

For the convenience of the reader we include a proof of (1.7) in the one variable case (the general case is similar, but notationally messy):

Since the binomial theorem holds in a commutative ring, we have

$$
\begin{aligned}
& p(X)=\sum_{k=0}^{m} c_{k} X^{k}=\sum_{k=0}^{m} c_{k}((X-a)+a)^{k} \\
& =\sum_{k=0}^{m} c_{k} \sum_{n=0}^{k}\left(\begin{array}{l}
k \\
n
\end{array}\right)(X-a)^{n} a^{k-n} \\
& =\sum_{n=0}^{m}(X-a)^{n} \sum_{k=n}^{m} \frac{k(k-1) \cdots(k-n+1) c_{k} a^{k-n}}{n !} \\
& =\sum_{n=0}^{m} \frac{p^{(n)}(a)}{n !}(X-a)^{n}
\end{aligned}
$$

We can use Proposition 1.3 to extend our "functional calculus" from polynomials to analytic functions:

1.4 Definition: If $f$ is analytic at $\left(x_{1}, \cdots, x_{k}\right)$, define

$$
f^{\diamond}\left(X_{1}, \cdots, X_{k}\right)=\sum_{\alpha} \frac{D_{\alpha} f\left(x_{1}, \cdots, x_{n}\right)}{\alpha_{1} ! \cdots \alpha_{k} !}\left(X_{1}-x_{1}\right)^{\diamond \alpha_{1}} \diamond \cdots \diamond\left(X-x_{k}\right)^{\diamond_{\alpha}}
$$


Note that since $\left(X_{1}-x_{1}\right)^{\diamond \alpha_{1}} \diamond \cdots \diamond\left(X-x_{k}\right)^{\diamond \alpha_{k}}=0$ when $|\alpha|=\alpha_{1}+\cdots+\alpha_{k}>|\Lambda|$, there are no convergence problems.

1.5 Example. Let us compute $\exp ^{\diamond}(X)$ when $X$ is a stochastic integral $X=\sum X(s) W(s)$ :

$$
\begin{aligned}
& \exp ^{\diamond}(X)=\sum_{n=0}^{\infty} \frac{1}{n !} X^{\diamond n}=\sum_{n=0}^{\infty} \frac{1}{n !}\left(\sum X(s) W(s)\right)^{\diamond n} \\
& =\sum_{n=0}^{\infty} \frac{1}{n !} \sum_{\substack{\left(s_{1}, \cdots, s_{n}\right) \\
s_{1}, \cdots, s_{n} \text { distinct }}} X\left(s_{1}\right) \cdot \ldots \cdot X\left(s_{n}\right) W\left(s_{1}\right) \cdot \ldots \cdot W\left(s_{n}\right) \\
& =\prod_{s \in \Lambda}(1+X(s) W(s))
\end{aligned}
$$

Recall that $W(s)=\sqrt{\mu(s)} \omega_{s}$. If $\mu(s)$ is very small (infinitesimal?), then

$$
\begin{aligned}
& \exp ^{\diamond}(X)=\prod_{s \in \Lambda}(1+X(s) W(s))=e^{\sum \ln \left(1+X(s) \sqrt{\mu(s)} \omega_{s}\right)} \\
& \approx e^{\sum X(s) W(s)-\frac{1}{2} \sum X^{2}(s) \mu(s)}=\frac{e^{\int X(s) d W(s)}}{e^{\frac{1}{2} \int X^{2}(s) d \mu(s)}}
\end{aligned}
$$

\section{Functional equations}

Let $a_{1}, a_{2}, \cdots, a_{N}$ be an enumeration of $\Lambda$. The space $\mathbb{C}^{\Lambda}$ of complex-valued functions over $\Lambda$ is isomorphic to $\mathbb{C}^{N}$ in an obvious way, and we shall move back and forth between the two spaces without further ado. Let

$$
F: \mathbb{C}^{N} \times \mathbb{C}^{k} \rightarrow \mathbb{C}^{N}
$$

be an analytic function, and let $Y_{1}, Y_{2}, \cdots, Y_{k}: \Omega \rightarrow \mathbb{C}$ be a (fixed) sequence of random variables with means $y_{1}, y_{2}, \cdots, y_{k}$. We want to study the relationship between solutions $X_{1}, X_{2}, \cdots, X_{N}$ of the stochastic functional equation

$$
F^{\diamond}\left(X_{1}, X_{2}, \cdots, X_{N}, Y_{1}, \cdots, Y_{k}\right)=0
$$

and solutions $x_{1}, x_{2}, \cdots, x_{N}$ of the deterministic equation

$$
F\left(x_{1}, x_{2}, \cdots, x_{N}, y_{1}, \cdots, y_{k}\right)=0
$$

Note that a solution $X=\left(X_{1}, \cdots, X_{N}\right)$ of (2.1) may be thought of as a stochastic process (or a random field) $X: \Lambda \times \Omega \rightarrow \mathbb{C}$. 
The idea is simple: we want to determine the Walsh decompositions $X_{i}=\sum X_{i}(A) \chi(A)$ of the solution of (2.1) by computing the Walsh decomposition of the left hand side of (2.1), and solving the ensuing equations for $X_{i}(A)$.

According to Proposition 1.3, the Walsh decomposition of the i-th component of $F^{\diamond}\left(X_{1}, \cdots, X_{N}, Y_{1}, \cdots, Y_{k}\right)$ is

$$
\begin{aligned}
& F_{i}^{\diamond}\left(X_{1}, \cdots, X_{N}, Y_{1}, \cdots, Y_{k}\right)= \\
& F_{i}\left(x_{1}, \cdots, x_{N}, y_{1}, \cdots, y_{k}\right) \\
& +\sum_{A \neq \emptyset}\left\{\sum_{\alpha} \sum_{<\left\{A_{j}^{i}\right\}>\in \mathcal{P}_{\alpha}(A)} D_{\alpha} F_{i}\left(x_{1}, \cdots, x_{N}, y_{1}, \cdots, y_{k}\right) X_{1}\left(A_{1}^{(1)}\right) \cdots\right. \\
& \left.X_{1}\left(A_{\alpha_{1}}^{(1)}\right) \cdots Y_{k}\left(A_{\alpha_{N+k}}^{(N+k)}\right)\right\} \chi(A)
\end{aligned}
$$

By uniqueness of the Walsh decomposition, this means that $X_{1}, \cdots, X_{N}$ is a solution of (2.1) if and only if the following set of equations is satisfied:

$$
F_{i}\left(x_{1}, \cdots, x_{N}, y_{1}, \cdots, y_{k}\right)=0
$$

for $A=\emptyset$, and for all other $A$,

$$
\sum_{\alpha} \sum_{<\left\{A_{j}^{i}\right\}>\in \mathcal{P}_{\alpha}(A)} D_{\alpha} F_{i}\left(x_{1}, \cdots, x_{N}, y_{1}, \cdots, y_{k}\right) X_{1}\left(A_{1}^{(1)}\right) \cdots X_{1}\left(A_{\alpha_{1}}^{(1)}\right) \cdots Y_{k}\left(A_{\alpha_{N}+k}^{(N+k)}\right)=0
$$

This system is not as complicated as it may look at first glance. To see why, assume that we have determined $X_{1}(B), \cdots, X_{N}(B)$ for all proper subsets $B$ of $A$. Then almost all the terms in (2.5) are known; in fact, the only unknown quantities are the $X_{j}(A)$-terms which appear when $D_{\alpha}=\frac{\partial}{\partial x_{j}}$. This structure becomes more evident if we rewrite (2.5) as

$$
\begin{aligned}
& \sum_{j=1}^{N} \frac{\partial F_{i}}{\partial x_{j}}\left(x_{1}, \cdots, x_{N}, y_{1}, \cdots, y_{k}\right) X_{j}(A) \\
& =-\sum_{l=1}^{k} \frac{\partial F_{i}}{\partial y_{l}}\left(x_{1}, \cdots, x_{N}, y_{1}, \cdots, y_{k}\right) Y_{l}(A) \\
& -\sum_{\substack{\alpha \\
|\alpha|>1}} \sum_{\left\{A_{j}^{i}\right\}>\in \mathcal{P}_{\alpha}(A)} D_{\alpha} F_{i}\left(x_{1}, \cdots, x_{N}, y_{1}, \cdots, y_{k}\right) X_{1}\left(A_{1}^{(1)}\right) \cdot \ldots \cdot Y_{k}\left(A_{\alpha_{N}+k}^{(N+k)}\right),
\end{aligned}
$$

where all terms on the right hand side are known. Recalling that what we have is really a system of equations - one for each value of the index $i$ - we see that our unknown quantities are the solutions of a very simple, linear system.

The strategy for finding the Walsh components of $X=\left(X_{1}, X_{2}, \cdots, X_{N}\right)$ is now clear. We first solve the deterministic equations (2.4). Using these solutions as an input, we can then 
solve (2.6) for all singletons $A=\left\{a_{i}\right\}$. With these solutions we can solve (2.6) for all two element sets $A=\left\{a_{i}, a_{j}\right\}$, and so on. We sum up our findings in the following theorem.

2.1 Theorem. Assume that $F: \mathbb{C}^{N} \times \mathbb{C}^{k} \rightarrow \mathbb{C}^{N}$ is an analytic function, and let $Y_{1}, \cdots, Y_{k}$ : $\Omega \rightarrow \mathbb{C}$ be random variables with expectations $y_{1}, \cdots, y_{k}$ respectively. Assume also that the Jacobian matrix

$$
J F\left(x_{1}, \cdots, x_{N}, y_{1}, \cdots, y_{k}\right)=\left(\begin{array}{cccc}
\frac{\partial F_{1}}{\partial x_{1}} & \frac{\partial F_{1}}{\partial x_{2}} & \cdots & \frac{\partial F_{1}}{\partial x_{N}} \\
\vdots & & & \\
\frac{\partial F_{N}}{\partial x_{1}} & \frac{\partial F_{N}}{\partial x_{2}} & \cdots & \frac{\partial F_{N}}{\partial x_{N}}
\end{array}\right)
$$

is nonsingular. Then there is a one-to-one correspondence between solutions $x_{1}, \cdots, x_{N}$ of the deterministic equation

$$
F\left(x_{1}, \cdots, x_{N}, y_{1}, \cdots, y_{k}\right)=0
$$

and solutions $X_{1}, \cdots, X_{N}$ of the stochastic equation

$$
F^{\diamond}\left(X_{1}, \cdots, X_{N}, Y_{1}, \cdots, Y_{k}\right)=0
$$

Given a solution $x=\left(\begin{array}{c}x_{1} \\ \vdots \\ x_{N}\end{array}\right)$ of (2.8), the corresponding solution $X=\left(\begin{array}{c}X_{1} \\ \vdots \\ X_{N}\end{array}\right)$ of (2.9) is given by the following hierarchy of equations:

$$
X(\emptyset)=x
$$

and if $X(B)$ has been determined for all proper subsets of $A$, then $X(A)$ is given by

$$
\begin{aligned}
& X(A)=-J F\left(x_{1}, \cdots, x_{N}, y_{1}, \cdots, y_{k}\right)^{-1}\left[\sum_{l=1}^{k} \frac{\partial F}{\partial y_{l}}\left(x_{1}, \cdots, y_{k}\right) Y_{l}(A)\right. \\
& \left.+\sum_{\substack{\alpha \\
|\alpha|>1}} \sum_{<\left\{A_{j}^{i}\right\}>\in \mathcal{P}_{\alpha}(A)} D_{\alpha} F\left(x_{1}, \cdots, y_{k}\right) X_{1}\left(A_{1}^{(1)}\right) \cdot \ldots \cdot Y_{k}\left(A_{\alpha_{N+k}}^{(N+k)}\right)\right]
\end{aligned}
$$

As an immediate consequence of the argument above, we have the following corollary:

2.2 Corollary: Assume that $Y_{1}, \cdots, Y_{k}$ and $Z_{1}, \cdots, Z_{k}$ are two sequences of random variables with the same expectations (i.e. $y_{i}=z_{i}$ for all $i$ ). Fix a solution $x_{1}, \cdots, x_{N}$ of the equation

$$
F\left(x_{1}, \cdots, x_{N}, y_{1}, \cdots, y_{k}\right)=0
$$


and let $X_{1}, \cdots, X_{N}$ and $\hat{X}_{1}, \cdots, \hat{X}_{N}$ be the corresponding solutions of

$$
F^{\diamond}\left(X_{1}, \cdots, X_{N}, Y_{1}, \cdots, Y_{k}\right)=0
$$

and

$$
F^{\diamond}\left(\hat{X}_{1}, \cdots, \hat{X}_{N}, Z_{1}, \cdots, Z_{k}\right)=0
$$

respectively. If $Y(B)=Z(B)$ for all $B \subseteq A$, then $X(A)=\hat{X}(A)$.

In the next two sections we shall take a brief look at some applications of the results above. We emphasize that these sections are intended primarily as illustrations of the scope of the method and not as systematic expositions of new theory.

\section{Discrete Itô-Wick equations}

In this section we let

$$
\Lambda=\{0,1,2, \cdots, N\}
$$

and consider equations of the form

$$
\begin{aligned}
& X(0)=X_{0} \in \mathbb{C} \\
& X(n+1)=X(n)+G^{\diamond}(X(n), n) \Delta t+H^{\diamond}(X(n), n) \diamond \omega(n) \sqrt{\Delta t}
\end{aligned}
$$

where we think of $\Delta t$ as a "small", real number. Obviously, (3.1) is a discrete version of the continuous Itô-Wick equation

$$
X(t)=X_{0}+\int_{0}^{t} G^{\diamond}(X(s), s) d s+\int_{0}^{t} H^{\diamond}(X(s), s) \diamond d B(s)
$$

Equation (3.1) has a unique solution obtained by induction, and our aim is to describe this solution in terms of the general machinery developed above.

If we define $F: \mathbb{C}^{N+1} \times \mathbb{C}^{N} \rightarrow \mathbb{C}^{N+1}$ componentwise by

$$
F_{0}\left(x_{0}, \cdots, x_{N}, y_{0}, \cdots, y_{N-1}\right)=x_{0}-X_{0}
$$

and, for $i>0$,

$$
\begin{aligned}
& F_{i}\left(x_{0}, \cdots, x_{N}, y_{0}, \cdots, y_{N-1}\right)=x_{i}-x_{i-1}-G\left(x_{i-1}, i-1\right) \Delta t \\
& -H\left(x_{i-1}, i-1\right) y_{i-1} \sqrt{\Delta t},
\end{aligned}
$$


we see that (3.1) is equivalent to

$$
F^{\diamond}(X(0), \cdots, X(N), \omega(0), \cdots, \omega(N-1))=0
$$

which is exactly the kind of equation we studied in the preceding section.

In order to apply Theorem 2.1, we must first compute the Jacobian $J F\left(x_{0}, \cdots, y_{N-1}\right)$ and its inverse. A simple calculation yields

$$
J F\left(x_{0}, \cdots, x_{N}, y_{0}, \cdots, y_{N-1}\right)=\left(\begin{array}{cccccc}
1 & 0 & 0 & \cdots & & 0 \\
-a_{1} & 1 & 0 & & & \vdots \\
0 & -a_{2} & 1 & 0 & & \vdots \\
0 & 0 & -a_{3} & 1 & & \vdots \\
\vdots & \vdots & & \ddots & \ddots & \vdots \\
0 & 0 & \cdots & & -a_{N} & 1
\end{array}\right)
$$

where

$$
a_{i}=1+G^{\prime}\left(x_{i-1}, i-1\right) \Delta t+H^{\prime}\left(x_{i-1}, i-1\right) y_{i-1} \sqrt{\Delta t}
$$

(all derivatives will be with respect to the first variable). Since in our case $y_{i}=E(\omega(i))=0$, we simply get

$$
a_{i}=1+G^{\prime}\left(x_{i-1}, i-1\right) \Delta t \text {. }
$$

The inverse of (3.6) is

$$
J F\left(x_{0}, \cdots, y_{N-1}\right)^{-1}=\left(\begin{array}{cccccc}
1 & 0 & \cdots \cdots & & & 0 \\
a_{1} & 1 & & & & \vdots \\
a_{1} a_{2} & a_{2} & 1 & & \vdots \\
a_{1} a_{2} a_{3} & a_{2} a_{3} & a_{3} & 1 & & \vdots \\
\vdots & & & \ddots & \ddots & \vdots \\
a_{1} a_{2} \cdots a_{N} & \cdots \cdots & & & a_{N} & 1
\end{array}\right)
$$

We can now use Theorem 2.1 to compute the Walsh decomposition

$$
X_{n}=x_{n}+\sum_{A \neq \emptyset} X_{n}(A)
$$

of the solution of (3.1). The deterministic part $x_{n}$ is simply the solution of the equation

$$
\begin{aligned}
& x_{0}=X_{0} \\
& x_{n+1}=x_{n}+G\left(x_{n}, n\right) \Delta t
\end{aligned}
$$


obtained by "turning off" the noise in (3.1). If $X_{m}(B)$ is known for all $B \subset A$ and all $m \in \Lambda$, then $X_{n}(A)$ is given by equation (2.7). In the present setting this equation simplifies substantially as many of the partial derivatives and most of the $Y_{l}(A)$-terms are zero, and after a few rearrangements, we end up with

$$
\begin{aligned}
& X_{n}(A)= \\
& =\sum_{m=0}^{n-1} \prod_{i=m+2}^{n} a_{i} \sum_{k=2}^{|A|} G^{(k)}\left(x_{m}, m\right) \sum_{\left\{A_{1}, \cdots, A_{k}\right\} \in \mathcal{P}_{k}(A)} X_{m}\left(A_{1}\right) \cdots X_{m}\left(A_{k}\right) \Delta t+ \\
& +\sum_{\substack{m \in A \\
m<n}} \prod_{i=m+2}^{n} a_{i} \sum_{l=0}^{|A|-1} H^{(l)}\left(x_{m}, m\right) \sum_{\left\{B_{1}, \cdots, B_{l}\right\} \in \mathcal{P}_{l}(A \backslash\{m\})} X_{m}\left(B_{1}\right) \cdot \ldots \cdot X_{m}\left(B_{l}\right) \sqrt{\Delta t}
\end{aligned}
$$

where we have used the conventions that $\prod_{i=n+1}^{n} a_{i}=1$ and that

$$
\sum_{\left\{B_{1}, \cdots, B_{0}\right\} \in \mathcal{P}_{0}(A \backslash\{m\})} X_{m}\left(B_{1}\right) \cdot \ldots \cdot X_{m}\left(B_{0}\right)= \begin{cases}1 & \text { if } A=\{m\} \\ 0 & \text { otherwise }\end{cases}
$$

Equation (3.12) may be simplified further. A simple induction argument on the size of $A$ shows that $X_{n}(A)=0$ unless $n$ is larger than all the elements in $A$ (this is also an immediate consequence of the fact that $X$ is a nonanticipating process). If we write $[A]$ for the largest element in $A$, then (3.12) reduces to

$$
\begin{aligned}
& X_{n}(A)= \\
& =\sum_{m=[A]+1}^{n-1} \prod_{i=m+2}^{n} a_{i} \sum_{k=2}^{|A|} G^{(k)}\left(x_{m}, m\right) \sum_{\left\{A_{1}, \cdots, A_{k}\right\} \in \mathcal{P}_{k}(A)} X_{m}\left(A_{1}\right) \cdot \ldots \cdot X_{m}\left(A_{k}\right) \Delta t+ \\
& +\prod_{i=[A]+2}^{n} a_{i} \sum_{l=0}^{|A|-1} H^{(l)}\left(x_{[A]},[A]\right) \sum_{\left\{B_{1}, \cdots, B_{l}\right\} \in \mathcal{P}_{l}(A-\{[A]\})} X_{[A]}\left(B_{1}\right) \cdot \ldots \cdot X_{[A]}\left(B_{l}\right) \sqrt{\Delta t}
\end{aligned}
$$

when $n>[A]$ (here $\mathcal{P}_{k}(A)$ denotes the set of all partitions of $A$ with $k$ partition classes). This is still quite abstract, and it will be informative to work out what $X_{n}(A)$ is when $A$ is a one or two element set. For a singleton $\{A\}=j$, we get immediately from (3.13) that

$$
X_{n}(\{j\})= \begin{cases}0 & \text { if } n \leq j \\ \prod_{i=j+2}^{n} a_{i} H\left(x_{j}, j\right) \sqrt{\Delta t} & \text { if } n>j\end{cases}
$$

Summing over all $j$, we see that the "first order" contribution to the Walsh chaos is

$$
\sum_{j=0}^{N} X_{n}(\{j\}) \chi\{j\}=\sum_{j=0}^{N-1} \prod_{i=j+2}^{n} a_{i} H\left(x_{j}, j\right) W(j)
$$


where $W(j)=\dot{\chi}\{j\} \sqrt{\Delta t}$ is white noise. Since

$$
\prod_{i=j+2}^{n} a_{i}=\prod_{i=j+1}^{n-1}\left(1+G^{\prime}\left(x_{i}, i\right) \Delta t\right) \approx e^{\sum_{i=j+1}^{n-1} G^{\prime}\left(x_{i}, i\right) \Delta t},
$$

the continuous counterpart of the right hand side of (3.15) is

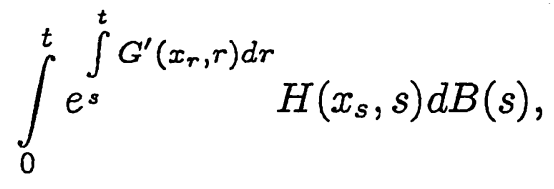

which "ought to be" the first order contribution to the Wiener-Itô chaos representation of the solution to the stochastic differential eqution (3.2).

Turning to two element sets $A=\{p, q\}, p<q$, we see from (3.13) that when $n>q$, then

$$
\begin{aligned}
& X_{n}\{p, q\}=\sum_{m=q+1}^{n-1} \prod_{i=m+2}^{n} a_{i} G^{(2)}\left(x_{m}, m\right) X_{m}(\{p\}) X_{m}(\{q\}) \Delta t \\
& +\prod_{i=q+2}^{n} a_{i} H^{\prime}\left(x_{q}, q\right) X_{q}(\{p\}) \sqrt{\Delta t}
\end{aligned}
$$

Using (3.14) to compute $X_{m}(\{p\}), X_{m}(\{q\})$ and $X_{q}(\{p\})$, and substituting the results into (3.17), we get

$$
\begin{aligned}
& X_{n}\{p, q\}=\prod_{i=p+2}^{n} a_{i} H\left(x_{p}, p\right) \prod_{i=q+2}^{n} a_{i} H\left(x_{q}, q\right) \sum_{m=q+1}^{n-1} \prod_{i=m+2}^{n} a_{i} G^{(2)}\left(x_{m}, m\right) \Delta t^{2} \\
& +\prod_{i=q+2}^{n} a_{i} H^{\prime}\left(x_{q}, q\right) \prod_{i=p+2}^{q} a_{i} H\left(x_{p}, p\right) \Delta t
\end{aligned}
$$

when $n>q$, and $X_{n}\{p, q\}=0$ when $n \leq q$. Summing over all $p$ and $q$, we get the following expression for the second order contribution to the Walsh chaos:

$$
\begin{aligned}
& \sum_{q=0}^{n-1} \sum_{p=0}^{q-1} \prod_{i=p+2}^{n} a_{i} H\left(x_{p}, p\right) \prod_{i=q+2}^{n} a_{i} H\left(x_{q}, q\right)\left[\sum_{m=q+1}^{n-1} \prod_{i=m+2}^{n} a_{i} G^{(2)}\left(x_{m}, m\right) \Delta t\right] W(p) W(q) \\
& +\sum_{q=0}^{n-1} \sum_{p=0}^{q-1} \prod_{i=p+2}^{q} a_{i} H\left(x_{p}, p\right) \prod_{i=q+2}^{n} a_{i} H\left(x_{q}, q\right) W(p) W(q)
\end{aligned}
$$

The continuous counterpart of this expression is

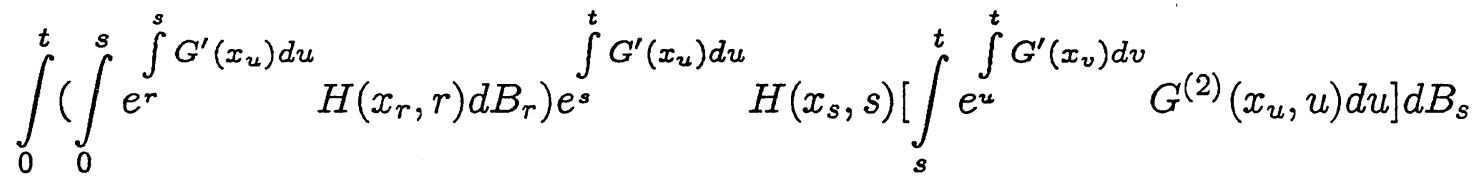

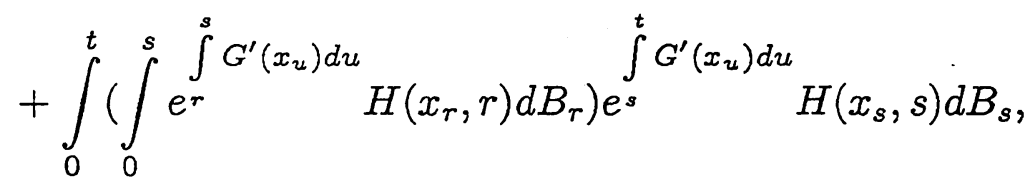


which should therefore be the second order component of the solution of (3.2).

In principle we can continue in this way to obtain higher order components, but in practice the computations soon become unwieldy; the third order term is a sum of ten iterated integrals and can still be written down quite easily, but the fourth order component has 82 terms!

An alternative approach to (continuous) Itô-Wick equations was developed in [3] and [4]. At the present stage it is not clear to us what the relationship between these two methods is; in both cases the original stochastic equation is replaced by a family of deterministic equations, but these deterministic equations are not the same in the two approaches! The advantage of the method developed in the present paper is that the only nonlinear equation we have to solve is the one obtained by turning off the noise in the given stochastic equation; the main disadvantage is that the linear equations which we also have to solve, interact in a quite complicated way. In the Hermite transform approach of [3] and [4] the nonlinear equation is usually more difficult to solve, but there is no coupling between the equations.

\section{Discrete Schrödinger operators with a random potential}

Fix a "small", positive number $\Delta x$, and let $\Lambda$ be a finite subset of the lattice

$$
\Gamma=\left\{\left(k_{1} \Delta x, \cdots, k_{d} \Delta x\right):\left(k_{1}, \cdots, k_{d}\right) \in \mathbb{Z}\right\}
$$

The interior $\Lambda^{\circ}$ of $\Lambda$ consists of those elements in $\Lambda$ whose nearest neighbors all belong to $\Lambda$, and the boundary $\partial A$ is the rest, i.e. $\partial \Lambda=\Lambda \backslash \Lambda^{\circ}$. The discrete Laplacian $\Delta$ on $\Lambda$ is defined by

$$
\Delta u(i)=\frac{1}{\Delta x^{2}}\left(u(i)-\frac{1}{2 d} \sum_{j \in N(i)} u(j)\right)
$$

for all $i \in \Lambda^{\circ}$, where $N(i)$ is the set of nearest neighbors of $i$.

For each $i \in \Lambda$, let $Y_{i}, Z_{i}: \Omega \rightarrow \mathbb{R}$ be random variables with expectation $y_{i}, z_{i}$, respectively, and assume that $y_{i}$ is positive for all $i \in \Lambda^{\circ}$. We are interested in the boundary value problem

$$
\begin{aligned}
& -\Delta X(i)+Y_{i} \diamond X(i)=Z_{i} \quad \text { for } i \in \Lambda^{\circ} \\
& X(i)=Z_{i} \text { for } i \in \partial \Lambda
\end{aligned}
$$

If we introduce a function

$$
F: \mathbb{C}^{\Lambda} \times \mathbb{C}^{\Lambda} \times \mathbb{C}^{\Lambda} \rightarrow \mathbb{C}^{\Lambda}
$$

by

$$
\begin{aligned}
& F_{i}\left(x_{1}, \cdots, x_{|\Lambda|}, y_{1}, \cdots, y_{|\Lambda|}, z_{1}, \cdots, z_{|\Lambda|}\right)= \\
& =-\frac{1}{\Delta x^{2}}\left(x_{i}-\frac{1}{2 d} \sum_{j \in N(i)} x_{j}\right)+y_{i} x_{i}-z_{i} \\
& =-\Delta x(i)+y_{i} x(i)-z_{i}
\end{aligned}
$$


for $i \in \Lambda^{\circ}$, and

$$
F_{i}\left(x_{1}, \cdots, z_{|\Lambda|}\right)=x_{i}-z_{i}
$$

for $i \in \partial \Lambda$, we see that (4.2) is equivalent to

$$
F^{\diamond}\left(X_{1}, \cdots, Z_{|\Lambda|}\right)=0 .
$$

The Jacobian of $F$ is just (the matrix corresponding to) the discrete Schrödinger operator $-\Delta+y$ with boundary conditions $z$, and since $y$ is positive, $-\Delta+y$ has an inverse $G$.

We are now ready to apply Theorem 2.1 . If

$$
X_{i}=x_{i}+\sum_{A \neq \emptyset} X_{i}(A) \chi(A)
$$

is the Walsh expansion of the solution of (4.2), then the deterministic part $x$ is simply the solution of the boundary value problem

$$
\begin{aligned}
& -\Delta x(i)+y_{i} x_{i}=z_{i} \quad \text { for } i \in \Lambda^{\circ} \\
& x_{i}=z_{i} \quad \text { for } i \in \partial \Lambda,
\end{aligned}
$$

and hence

$$
x=G z
$$

Since almost all partial derivatives of $F$ are zero, the recurrence relation (2.7) is particularly simple in this case; in fact

$$
X_{i}(A)=-\sum_{j \in \Lambda} G(i, j)\left[x_{j} Y_{j}(A)-Z_{j}(A)+\sum_{\substack{\subsetneq B \subsetneq \\ B}} X_{j}(B) Y_{j}(A \backslash B)\right] .
$$

If we are willing to simplify the problem further, we can use (4.8) to obtain an explicit expression for $X_{i}(A)$. Let us assume that $Z$ is deterministic (i.e. $Z_{i}=z_{i}$ for all $i$ ), and that $Y$ has the form $Y_{i}=y_{i}+\tilde{Y}_{i} \chi(i)$, where $\tilde{Y} \in \mathbb{R}$. Then (4.8) turns into

$$
X_{i}\{j\}=-G(i, j) x_{j} \tilde{Y}_{j}
$$

when $A$ is a singleton, and

$$
X_{i}(A)=-\sum_{j \in A} G(i, j) X_{j}(A \backslash\{j\}) \tilde{Y}_{j}
$$

when $A$ has more than one element. If we play around with these two formulas, we are led to the expression

$$
\begin{aligned}
& X_{i}\left\{j_{1}, \cdots, j_{k}\right\}= \\
& =(-1)^{k} \tilde{Y}_{j 1} \cdot \ldots \cdot \tilde{Y}_{j k} \sum_{\sigma} x_{j_{\sigma(k)}} G\left(i, j_{\sigma(1)}\right) G\left(j_{\sigma(1)}, j_{\sigma(2)}\right) \cdot \ldots \cdot G\left(j_{\sigma(k-1)}, j_{\sigma(k)}\right)
\end{aligned}
$$


where the sum is over all permulation $\sigma$ of the set $\{1,2, \cdots, k\}$.

To prove (4.11) by induction, first observe that by (4.9) the formula holds for $k=1$. Assume next that the formula holds for all sets of cardinality $k$, and let $\left\{j_{1}, \cdots, j_{k+1}\right\}$ be a set of cardinality $k+1$. By (4.10)

$$
X_{i}\left\{j_{1}, \cdots, j_{k+1}\right\}=-\sum_{l=1}^{k+1} G\left(i, j_{l}\right) X_{j_{l}}\left(j_{1}, \cdots, \hat{j}_{l}, \cdots, j_{k+1}\right) \tilde{Y}_{j_{l}}
$$

where the hat above $j_{l}$ means that $j_{l}$ is not part of the expression. Using the induction hypothesis, we get

$$
\begin{aligned}
& X_{i}\left\{j_{1}, \cdots, j_{k+1}\right\}=(-1)^{k+1} \tilde{Y}_{j_{1}} \cdot \ldots \cdot \tilde{Y}_{j_{k+1}} \times \\
& \times \sum_{l=1}^{k+1} G\left(i, j_{l}\right) \sum_{\sigma_{l}} x_{j_{\sigma_{l}(k)}} G\left(j_{l}, j_{\sigma_{2}(1)}\right) \cdot \ldots \cdot G\left(j_{\sigma_{l}(k-1)}, j_{\sigma_{l}(k)}\right)
\end{aligned}
$$

where for each $l \sigma_{l}$ runs over all permutations of the set $\{1, \cdots, \hat{l}, \cdots, k+1\}$. By rearranging (4.12) slightly, we get the expression we are looking for, and hence (4.11) is proved.

The full Walsh decomposition of $X$ can now be written as

$$
X_{i}=x_{i}+\sum_{k=1}^{|\Lambda|}(-1)^{k} \sum_{\left(j_{1}, \cdots, j_{k}\right) \in \Lambda^{k}} x_{j_{k}} G\left(i, j_{1}\right) \cdot \ldots \cdot G\left(j_{k-1}, j_{k}\right) \prod_{l=1}^{k} \tilde{Y}_{l} \chi\left\{j_{1}, \cdots, j_{k}\right\}
$$

For simplicity we have in this section worked with the Laplace operator, but it is clear that the general method applies equally well to any other operator - even nonlinear ones as long as we are able to "solve" the corresponding deterministic equation.

In [2] we have developed a different approach to problems of this kind (based on extensions of the Hermite transform methods from [3] and [4]). As with the ordinary differential equations in Section 3, the two methods lead to different kind of computations, and the relationship between the two approaches is not at all clear.

\section{Final remarks}

The purpose of this paper has been to point out the close relationship between stochastic Wick equations and their associated determinstic equations in the discrete case. Since nobody is very much interested in discrete Wick calculus for its own sake, our real aim is to extend our approach to the continuous case, but this leads to difficult convergence problems which we have so far not studied in any depth. We are particularly interested in understanding the relationship between the approach in this paper and the Hermite transform methods developed in [2], [3] and [4]. 


\section{References}

1. T. Hida, H.-H. Kuo, J. Potthoff, and L. Streit: White noise. An infinite dimensional calculus. Book in preparation.

2. H. Holden, T. Lindstrøm, B. Øksendal, J. Ubøe and T.-S. Zhang: Stochastic boundary value problems. A white noise functional approach, Submitted to Probab. Th. Related Fields.

3. T. Lindstr $\varnothing \mathrm{m}$, B. $\varnothing \mathrm{ksendal}$, and J. Ubøe: Stochastic differential equations involving positive noise, in N. Bingham and M.T. Barlow (eds.) "Stochastic Analysis", Cambridge University Press, Cambridge, 1991, 261-303.

4. T. Lindstrøm, B. Øksendal, and J. Ubøe: Wick multiplication and Itô-Skorohod stochastic differential equations, to appear in S. Albeverio et al. (eds): Ideas and methods in mathematical analysis, stochastics, and applications, Cambridge University Press.

5. P.A. Meyer: A finite approximation to Boson Fock space, in S. Albeverio et al. (eds): Stochastic processes in classical and quantum systems, Springer-Verlag, 1986, 405410.

6. P.A. Meyer: Fock space and probability theory, in S. Albeverio et al. (eds): Stochastic processes - mathematics and physics, LNM 1250, Springer-Verlag 1987, 160-170. 